\title{
Nicotinamide: a novel treatment for age-related macular degeneration?
}

\author{
Peter D. Westenskow \\ Department of Ophthalmology, Baylor College of Medicine, Houston, USA \\ Correspondence to: Peter D. Westenskow, PhD. Baylor College of Medicine, 6565 Fannin St, NA-520 Houston, TX 77030, USA. \\ Email: peter.westenskow@bcm.edu. \\ Provenance: This is an invited Editorial commissioned by Editor-in-Chief Zhizhuang Joe Zhao (Pathology Graduate Program, University of \\ Oklahoma Health Sciences Center, Oklahoma City, USA). \\ Comment on: Saini JS, Corneo B, Miller JD, et al. Nicotinamide ameliorates disease phenotypes in a human iPSC model of age-related macular \\ degeneration. Cell Stem Cell 2017;20:635-647.e7.
}

Received: 20 September 2017; Accepted: 28 September 2017; Published: 27 October 2017.

doi: $10.21037 /$ sci.2017.10.01

View this article at: http://dx.doi.org/10.21037/sci.2017.10.01

Saini et al. recently reported in Cell Stem Cell that nicotinamide can suppress the expression of a broad set of genes in induced pluripotent stem cell-derived retinal pigment epithelium (iPS-RPE) cells that may contribute to age-related macular degeneration (AMD) (1). There are ample reasons to be excited about their findings, and many opportunities for future work. In this commentary, I will begin by providing background about the etiology of AMD before outlining some of the challenges of studying AMD using iPS-RPE. With that context in place, I will describe which elements of the Saini et al. study I find most exciting, and discuss potential future directions.

\section{The outer retina-a finely tuned machine}

The outer half of the retina consists of hard working cells and structures that convert light into neural signals through phototransduction. Rod and cone photoreceptors initiate phototransduction when activated by light, but cannot complete the process without help from the multifunctional retinal pigment epithelium (RPE) or without fuel. Energy substrates for the RPE and photoreceptors are provided by a highly dense and fenestrated extraretinal vascular plexus, the choriocapillaris, which surrounds the outer retina (Figure 1A).

The choriocapillaris, RPE cells, and photoreceptors are all uniquely remarkable. According to many accounts, the blood flow rate and the vascular permeability of the choriocapillaris is higher than anywhere in the body (2).
Oxygen and nutrients from the choriocapillaris are filtered and redistributed in the subretinal space through a pentalaminar matrix (Bruch's membrane) and by RPE cells. RPE also generate and secrete key vasculotrophic factors for choriocapillaris maintenance, deliver glucose to photoreceptors, recycle spent photopigments, absorb stray light, and phagocytose light damaged photoreceptor membranes to prevent oxidative stress (3). Photoreceptors can be activated by a single photon, and can respond appropriately to the wavelength and intensity of a light source. However, they are also extraordinarily metabolically demanding and inherently fragile (4). Maintaining the integrity and homeostasis of photoreceptors, RPE, and the choriocapillaris is imperative, and defects result in profound vision loss.

\section{Even finely tuned machines break down over time}

The prevalence of age-related vision loss should not be surprising considering the intense burdens placed on the choriocapillaris and RPE to support the remarkable metabolic demands of photoreceptors. In fact, AMD is a commonly occurring and leading cause of vision loss in industrialized countries. The global prevalence of AMD is steadily increasing and expected to reach epidemic proportions by the year 2040 when some experts estimate that 288 million people could be affected (5). To emphasize 

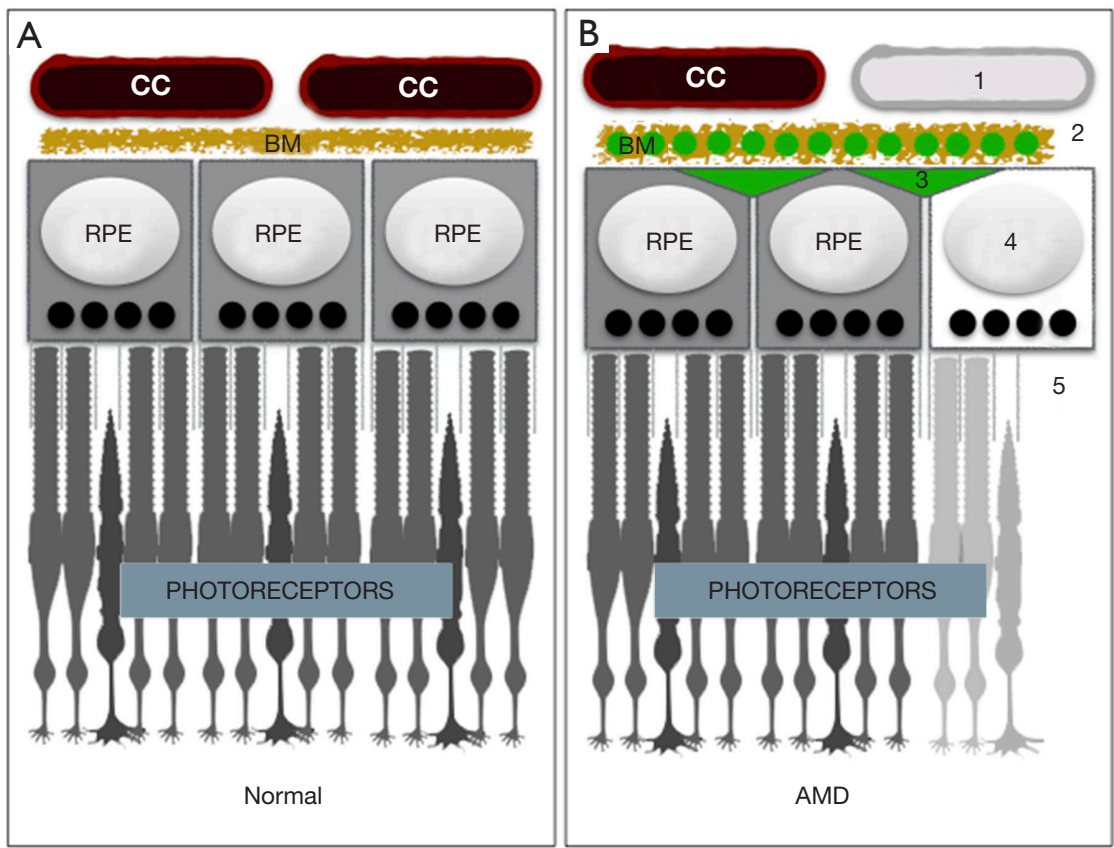

Figure 1 Anatomy of the outer retina and cardinal features of age-related macular degeneration (AMD). (A) The extraretinal choriocapillaris (CC) is divided from the retinal pigment epithelium (RPE) by Bruch's membrane (BM). RPE provide critical support for photoreceptors; (B) features of AMD, arbitrarily numbered top to bottom, are as follows: (1) choriocapillaris drop out; (2) BM with lipids (green circles) and reduced diffusibility; (3) formation of drusen that also limits diffusibility; (4) RPE dysfunction/death; and (5) photoreceptor atrophy. Choroidal neovascularization characteristic of wet AMD, is not shown.

the enormity of this problem, the population of the United States of America was 327 million as of September, 2017, based on United Nations Population Division estimates.

\section{AMD}

There are two forms of AMD: dry (geographic atrophy) and wet (neovascular or exudative AMD). In wet AMD, blood vessels from the choriocapillaris invade the space occupied by photoreceptors and damage the macula, which is responsible for color and high-acuity vision. Treatments for wet AMD include repeated intraocular injections of VEGF antagonists to slow blood vessel growth. Dry AMD also affects the macula, but is far more prevalent than wet AMD (90\% vs. $10 \%$ respectively), and no treatments exist. Cardinal features include accumulation of lipids and proteins within drusen and Bruch's membrane (6), RPE dysfunction/death, complementmediated attack and vaso-obliteration of the choriocapillaris (7), and photoreceptor atrophy (Figure 1B). However, the precise etiology of AMD is unclear $(7,8)$, and according to some experts it may be a spectrum of closely related diseases (9). Both genetic and environmental influences confer risk, but it is unclear which outer retinal components are affected first by the insult(s), and the order in which they are affected may differ between cases (8).

To gain new insights into AMD pathophysiology, Saini et al. generated iPS-RPE from AMD patients and agematched controls and looked for differences between the groups using gene, protein, and cytokine profiling assays. While they detected few differences between healthy and disease groups, they did make an interesting discovery: nicotinamide, which accelerates iPS-RPE differentiation and has been shown to prevent neurodegeneration in other systems $(10,11)$, can modulate a set of genes and proteins that may contribute to AMD in iPS-RPE. Not only did they identify a wide array of genes that nicotinamide modulates in iPS-RPE, they also showed that it is effective across several genetic backgrounds, including in AMD iPS-RPE harboring largely uncharacterized AMD mutations (12).

\section{The challenges of developing AMD models}

To fully appreciate the excitement generated from the Saini et al. study, and to avoid over-interpreting their findings, 


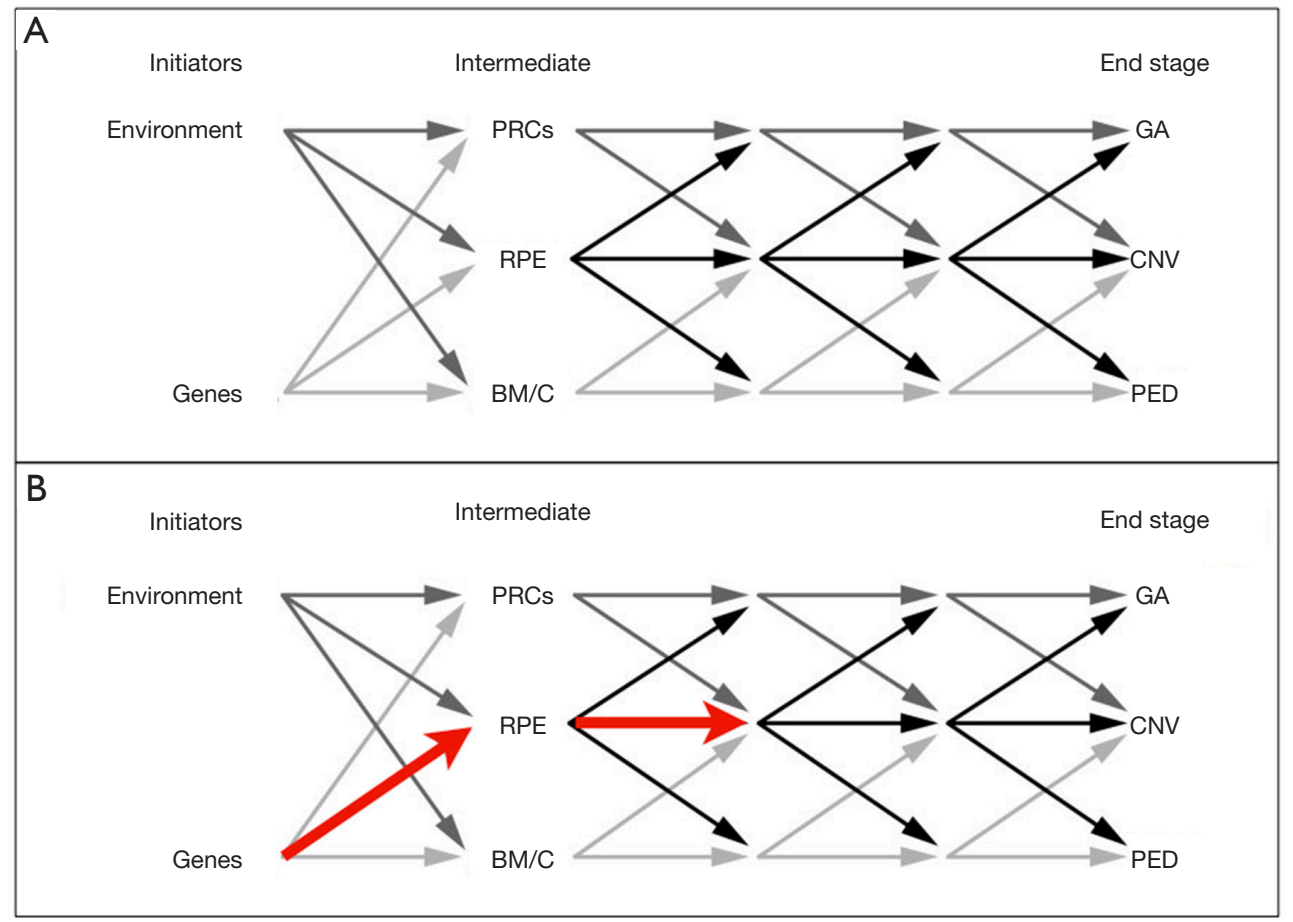

Figure 2 Putative disease pathways in AMD, and limitations of using iPS-RPE for AMD modeling. (A) Initiating factors combine and converge on photoreceptor cells (PRCs), RPE, Bruch's Membrane (BM), and/or the choroid (C). Changes to these components initiate a cascade of intermediate disease mechanisms. (B) Red lines mark the limited events from which data can be obtained from iPS-RPE. Only genetic contributions are interrogated, and downstream effects propagated through early and intermediate stages can only be predicted. Reproduced with modifications and permission of the American Society for Clinical Investigation, from Bird AC, JCI 2010 (8); permission conveyed through the Copyright Clearance Center Inc.

I will begin this section by briefly describing some of the complications of modeling AMD, by outlining some of the caveats of using patient-specific iPSCs to study AMD, and by explaining additional complications for using RPE in these types of studies.

\section{AMD is a multifactorial and complex disease with an unknown etiology}

Major problems facing AMD researchers are that normal age-related changes in the outer retina can be difficult to distinguish from AMD-related ones and a paucity of viable animal and cellular disease models $(13,14)$. Developing models is difficult since the initial events of AMD are caused by combinations of environmental and genetic factors that modify the choriocapillaris, Bruch's membrane, RPE, and/or photoreceptors, but never in any particular order $(8,13)$. Furthermore, in early and intermediate stages, changes in any of these components initiate a chain reaction that affects some or all the others (Figure 2A). Finally, even the final AMD events, geographic atrophy (GA), choroidal neovascularization (CNV), and pigment epithelial detachment (PED) are only partially understood (8).

The complicated genetics of AMD, and the expression patterns of AMD linked genes, also limits our ability to generate transgenic and cellular models. While genetic tests can be used to identify mutations that confer risk, the results of these tests cannot be used to accurately predict if a patient will get AMD or how the disease will progress. Genetic background can also influence the likelihood that a person will have a specific AMD phenotype and affect the age of onset and rate of progression (15). Consequently, modifying the same gene in RPE cells from different genetic backgrounds could produce graded or heterogeneous phenotypes. In addition, AMD linked genes do not have eye-specific expression patterns (15). Therefore, the primary event might occur outside the eye and be manifested secondarily in the outer retina. For these 
reasons, AMD models are difficult to generate and while transgenic and cellular models of AMD may yield valuable data in many instances, they could also yield very limited, or even misleading, information about the disease in others.

\section{Caveats of iPS-RPE based AMD disease modeling}

Examining iPS-RPE derived from AMD patients with known mutations may provide some insights into the etiology; however, these studies are frequently hampered by noisy datasets, may not represent physiological situations, and offer only a limited view into disease progression.

Many independent groups, including ours, have shown that RPE can be readily generated from human iPSCs that strongly resemble primary human RPE. However, many other independent groups have shown that the process of reprogramming somatic cells to iPSCs introduces epigenetic changes that can affect the cellular phenotype of iPSderived cells. In an excellent review from Sandoe et al. (16), the authors describe some of the sources of transcriptomic noise in iPSCs. They explain that noise increases when cells from different genetic backgrounds are compared, when multiple reprogramming methods are employed within sample sets, when iPSCs are derived from different tissue sources, and due to $\mathrm{X}$-linked inactivation. However, they also explain that these issues are not insurmountable; noise can be reduced, and sampling errors can be obviated, by using a large number of patient samples and/or by using isogenic cell-lines (in which that mutation was introduced in cells with identical genetic backgrounds) (see Sandoe et al. the first table for examples).

Besides noise, another caveat with iPS-RPE based AMD studies is that AMD mutations may induce inconsistent and variable phenotypes in RPE, and some observed effects may not be relevant, or recapitulated in vivo. Many iPS-RPE based AMD studies are based on the unlikely assumption that RPE are the first, or only, outer retina component affected by the mutation and that other environmental issues do not contribute to the phenotype. Environmental issues that may contribute include reduced oxygen/nutrient availability due to impaired diffusibility through confluent drusen and/or a "lipid wall" in Bruch's membrane (6); ischemia due to diffusibility issues and/or choriocapillaris drop-out, which we showed fundamentally alters gene expression and metabolic behaviors of RPE (17); and/or intraretinal vascular changes and hyperoxia due to photoreceptor degeneration (4). For example, if a patient's AMD mutations induced a primary defect in the choriocapillaris or Bruch's membrane potent enough to induce ischemia in RPE, examining iPS-RPE in vitro from that patient in normoxic conditions will yield very little, if any, relevant information about the patient's disease. Finally, we cannot measure the compounding effects of AMD mutations in RPE propagated through photoreceptors, Bruch's membrane, and the choriocapillaris across early and intermediate stages, we can only predict them. For these reasons, studies in patient derived iPS-RPE may only provide inconsistent and limited views of AMD pathogenesis (Figure 2B), and any data from iPS-RPE AMD studies must be interpreted carefully considering these constraints.

\section{Technical issues involving iPS-RPE}

There are also potential pitfalls to avoid when using iPSRPE. Serial passaging iPS-RPE lines induces phenotypic drift (18), iPS-RPE may not reach peak function until several months post-differentiation (19), and RPE have significantly different expression profiles when polarized (20). Therefore, when analyzing data from iPS-RPE, it is important to consider the number of passages of the lines used, how long the cells have been maintained in culture, and whether the RPE cells were grown on transwell filters to promote polarization.

\section{Nicotinamide suppresses signaling cascades in RPE that might contribute to AMD}

Despite the gloomy perspective I described in the previous section, iPS-RPE based AMD studies are important, can yield important data about AMD etiology and drugresponsiveness, and should be performed. For as daunting as iPS-RPE AMD disease modeling is, developing relevant animal AMD models is equally, or more, difficult. Saini et al. have shown that relevant and interesting data can be "squeezed" from in vitro models within the constraints explained in previous sections.

A major strength of their work, was the finding that nicotinamide can elicit broad effects on AMD RPE that could be predicted to cascade onto photoreceptors, Bruch's membrane, and the choriocapillaris. Using bioinformatics, they identified functionally related gene clusters affected by nicotinamide and sorted them according to KEGG and disease pathways. Notable hits from this analysis, which will be addressed in order, include the PI3-Akt signaling pathway, complement cascades, and ECM- 
receptor interactions. First, the PI3/Akt/mTOR pathway has been shown to regulate RPE metabolism (21), and stimulation of the insulin/mTOR pathway in murine retinas significantly retards photoreceptor atrophy (22). Second, variants in complement cascade genes including $C F H, C F I, C F B$, and $C 3$ are associated with $\mathrm{AMD}$, and complement-mediated attacks on the choriocapillaris might be a primary event in AMD pathogenesis (7). Third, ECM receptor interactions are critical for choriocapillaris, Bruch's membrane, and RPE crosstalk (23). In addition, Saini et al. used bioinformatics to predict nicotinamide-mediated changes in retinal degeneration, cardiomyopathy, and Alzheimer's disease pathways. These predicted connections are interesting since associations may exist between AMD and lipid-handling and cardiovascular factors (24), and multiple studies now link $A \beta$ with key stages of AMD progression (25).

Besides the predicted associations from RNA-seq data, the authors reported measured nicotinamide-mediated reductions in $C 3, A \beta-42, A P O E, A P O \mathcal{F}$, and VEGFA genes and their corresponding proteins that could be predicted to impact choriocapillaris and photoreceptor viability, lipid-handling in RPE, and Bruch's membrane diffusibility. Nicotinamide reduced $C 3$ mRNA by approximately $90 \%$, and secreted protein levels 6-7-fold. This is significant since complement inhibitors are being designed and tested in clinical trials for AMD (26). Subretinal injections of $A \beta_{42}$ in mice induce anatomical defects in RPE and pronounced photoreceptor atrophy (27). Finally, lipid-handling defects in RPE can result in lipid extrusion and Bruch's membrane thickening in mice (17), and ApoE and ApoJ are the most prevalent apolipoproteins detected in human drusen (28).

The observation that VEGF-A is suppressed by nicotinamide is the most difficult to interpret, especially since we do not know if the patients involved in this study had wet or dry AMD. If the patients had wet AMD, reducing VEGF might be beneficial, but reducing VEGF below threshold levels in dry AMD patients can exacerbate disease progression. In primates, single intravitreal injections of VEGF antagonists transiently defenestrate and induce vessel occlusion in the choriocapillaris (29).In mice, we showed that single anti-VEGF injections promote complement accumulation around retinal capillary beds that predispose them to vaso-obliteration (30), and genetic ablation of Vegfa in adult murine RPE results in severe choriocapillaris drop-out and profound cone dysfunction three and seven days-post-induction respectively $(17,31)$. Finally, chronic anti-VEGF injections can cause GA in wet
AMD patients (32). Therefore, if validated for human use, nicotinamide might be best employed as a therapy for wet AMD; however, care must be taken to not cause GA in wet AMD patients by over-antagonizing VEGF and causing damage to ocular vascular networks.

\section{Future directions}

The findings from Saini et al. open a door for several possible future studies: (I) establishing the physiological consequences of the nicotinamide-mediated mRNA and protein accumulation changes; (II) determining if nicotinamide is equally effective across other AMD iPSRPE lines with other disease associated mutations and genetic backgrounds; and (III) screening for novel drug candidates in the cell lines generated by Saini et al., and in others contributed by the scientific community.

Many of the effects on mRNA and protein expression elicited by nicotinamide must be confirmed and downstream events be examined to establish physiological relevance. The nicotinamide-mediated suppression of $\mathrm{C} 3$ is encouraging, but once $\mathrm{C} 3$ is secreted from a cell many downstream events must occur to generate membrane attack complexes. This applies to ApoE and ApoJ secretion as well; it is important to show if reduced expression of both proteins impacts drusen formation and Bruch's membrane thickening in physiological contexts, especially since drusen are very common in aging eyes and probably do not cause AMD. In fact, one or more drusen can be found in $95.5-98.8 \%$ of the general population, and risk for developing AMD is assessed on a case-by-case basis according to the type (hard or soft), location (in or outside the macula), and size of each druse (33). Whether nicotinamide prevents accumulation of the types of drusen that confer risk for AMD remains to be determined.

The amount of VEGF inhibition induced by nicotinamide must be established in vivo to determine which form of AMD to target. In addition, the amount of VEGF suppression by nicotinamide must be deemed safe for dry AMD patients before initiating clinical trials.

Finally, if we could generate an expansive collection of iPSRPE with known mutations (and complete clinical histories) that span multiple diverse genetic backgrounds, we could test novel therapeutic agents, and perhaps generate patient-specific drug cocktails to treat specific features of AMD.

\section{Summary and conclusions}

The outer retina consists of uniquely remarkable cells 
and structures that are responsible for phototransduction. However, phototransduction is metabolically expensive, and overworked photoreceptors, RPE, Bruch's membrane, and the choriocapillaris are prone to age-related changes. Changes in one member of the unit cascade onto other members of the unit throughout early and intermediate stages of AMD disease progression, cumulatively resulting in vision loss. Due to the genetic and etiological complexity of AMD, the discovery of drugs that exert broad effects on outer retina physiology might be the best solution, and nicotinamide has potential to be that type of drug. Nicotinamide can suppress accumulation of apolipoproteins, members of the complement cascade, and extracellular matrix receptors that may damage RPE, photoreceptors, Bruch's membrane, and/or the choriocapillaris. If those predictions are correct, and if the effects of nicotinamide can be shown to be physiologically relevant, nicotinamide could eventually be used treat some features of AMD in at least a subset of AMD patients.

\section{Acknowledgements}

I wish to acknowledge the excellent tutelage of Dr. Alan C. Bird (Professor Emeritus, Moorfield's Eye Hospital), and to thank Drs. John Mazzilli (UT Health) and Kevin Eade (the Lowy Medical Research Institute) for critically reading and improving this manuscript.

Funding: Funding sources include a core grant from the NIH to Baylor College of Medicine (2P30EY002520), and unrestricted grants to the Department of Ophthalmology at Baylor College of Medicine from the Research to Prevent Blindness (RPB) health organization.

\section{Footnote}

Conflicts of Interest: The author has no conflict of interest to declare.

\section{References}

1. Saini JS, Corneo B, Miller JD, et al. Nicotinamide Ameliorates Disease Phenotypes in a Human iPSC Model of Age-Related Macular Degeneration. Cell Stem Cell 2017;20:635-647.e7.

2. Sapieha P. Eyeing central neurons in vascular growth and reparative angiogenesis. Blood 2012;120:2182-94.

3. Strauss $\mathrm{O}$. The retinal pigment epithelium in visual function. Physiol Rev 2005;85:845-81.
4. Stone J, Maslim J, Valter-Kocsi K, et al. Mechanisms of photoreceptor death and survival in mammalian retina. Prog Retin Eye Res 1999;18:689-735.

5. Wong WL, Su X, Li X, et al. Global prevalence of age-related macular degeneration and disease burden projection for 2020 and 2040: a systematic review and meta-analysis. Lancet Glob Health 2014;2:e106-16.

6. Curcio CA, Johnson M, Rudolf M, et al. The oil spill in ageing Bruch membrane. Br J Ophthalmol 2011;95:1638-45.

7. Whitmore SS, Sohn EH, Chirco KR, et al. Complement activation and choriocapillaris loss in early AMD: implications for pathophysiology and therapy. Prog Retin Eye Res 2015;45:1-29.

8. Bird AC. Therapeutic targets in age-related macular disease. J Clin Invest 2010;120:3033-41.

9. Bird AC, Phillips RL, Hageman GS. Geographic atrophy: a histopathological assessment. JAMA Ophthalmol 2014;132:338-45.

10. Green KN, Steffan JS, Martinez-Coria H, et al. Nicotinamide restores cognition in Alzheimer's disease transgenic mice via a mechanism involving sirtuin inhibition and selective reduction of Thr231-phosphotau. J Neurosci 2008;28:11500-10.

11. Idelson M, Alper R, Obolensky A, et al. Directed differentiation of human embryonic stem cells into functional retinal pigment epithelium cells. Cell Stem Cell 2009;5:396-408.

12. Sun S, Li Z, Glencer P, et al. Bringing the age-related macular degeneration high-risk allele age-related maculopathy susceptibility 2 into focus with stem cell technology. Stem Cell Res Ther 2017;8:135.

13. Forest DL, Johnson LV, Clegg DO. Cellular models and therapies for age-related macular degeneration. Dis Model Mech 2015;8:421-7.

14. Guymer R, Bird AC. Age Changes in Bruch's Membrane and Related Structures. In: Ryan SJ, editor. Retina. 4th ed. Elsevier Mosby 2006. p. 598-614.

15. Gorin MB. Genetic insights into age-related macular degeneration: controversies addressing risk, causality, and therapeutics. Mol Aspects Med 2012;33:467-86.

16. Sandoe J, Eggan K. Opportunities and challenges of pluripotent stem cell neurodegenerative disease models. Nat Neurosci 2013;16:780-9.

17. Kurihara T, Westenskow PD, Gantner ML, et al. Hypoxiainduced metabolic stress in retinal pigment epithelial cells is sufficient to induce photoreceptor degeneration. Elife 2016;5:pii:e14319.

18. Singh R, Phillips MJ, Kuai D, et al. Functional analysis 
of serially expanded human iPS cell-derived rpe cultures. Invest Ophthalmol Vis Sci 2013;54:6767-78.

19. Westenskow PD, Moreno SK, Krohne TU, et al. Using flow cytometry to compare the dynamics of photoreceptor outer segment phagocytosis in iPS-derived RPE cells. Invest Ophthalmol Vis Sci 2012;53:6282-90.

20. Sonoda S, Sreekumar PG, Kase S, et al. Attainment of polarity promotes growth factor secretion by retinal pigment epithelial cells: relevance to age-related macular degeneration. Aging (Albany NY) 2009;2:28-42.

21. Zhao C, Yasumura D, Li X, et al. mTOR-mediated dedifferentiation of the retinal pigment epithelium initiates photoreceptor degeneration in mice. J Clin Invest 2011;121:369-83.

22. Punzo C, Kornacker K, Cepko CL. Stimulation of the insulin/mTOR pathway delays cone death in a mouse model of retinitis pigmentosa. Nat Neurosci 2009;12:44-52.

23. Benedicto I, Lehmann GL, Ginsberg M, et al. Concerted regulation of retinal pigment epithelium basement membrane and barrier function by angiocrine factors. Nat Commun 2017;8:15374.

24. Pennington KL, DeAngelis MM. Epidemiology of agerelated macular degeneration (AMD): associations with cardiovascular disease phenotypes and lipid factors. Eye Vis (Lond) 2016;3:34.

25. Ratnayaka JA, Serpell LC, Lotery AJ. Dementia of the eye: the role of amyloid beta in retinal degeneration. Eye (Lond) 2015;29:1013-26.

doi: $10.21037 /$ sci.2017.10.01

Cite this article as: Westenskow PD. Nicotinamide: a novel treatment for age-related macular degeneration? Stem Cell Investig 2017;4:86.
26. Holz FG, Schmitz-Valckenberg S, Fleckenstein M. Recent developments in the treatment of age-related macular degeneration. J Clin Invest 2014;124:1430-8.

27. Bruban J, Glotin AL, Dinet V, et al. Amyloid-beta(1-42) alters structure and function of retinal pigmented epithelial cells. Aging Cell 2009;8:162-77.

28. Li CM, Clark ME, Chimento MF, et al. Apolipoprotein localization in isolated drusen and retinal apolipoprotein gene expression. Invest Ophthalmol Vis Sci 2006;47:3119-28.

29. Peters S, Heiduschka P, Julien S, et al. Ultrastructural findings in the primate eye after intravitreal injection of bevacizumab. Am J Ophthalmol 2007;143:995-1002.

30. Keir LS, Firth R, Aponik L, et al. VEGF regulates local inhibitory complement proteins in the eye and kidney. J Clin Invest 2017;127:199-214.

31. Kurihara T, Westenskow PD, Bravo S, et al. Targeted deletion of Vegfa in adult mice induces vision loss. J Clin Invest 2012;122:4213-7.

32. Comparison of Age-related Macular Degeneration Treatments Trials (CATT) Research Group, Martin DF, Maguire MG, et al. Ranibizumab and bevacizumab for treatment of neovascular age-related macular degeneration: two-year results. Ophthalmology 2012;119:1388-98.

33. Sarks SH, Arnold JJ, Killingsworth MC, et al. Early drusen formation in the normal and aging eye and their relation to age related maculopathy: a clinicopathological study. $\mathrm{Br}$ J Ophthalmol 1999;83:358-68. 